International Journal of Modern Physics D

(C) World Scientific Publishing Company

\title{
The Gravitational Energy Problem for Cosmological Models in Teleparallel Gravity
}

\author{
S. C. Ulhoa \\ International Institute for Physics, \\ Universidade Federal do Rio Grande do Norte, Campus Universitário, Lagoa Nova. Natal-RN, \\ Brazil. P. O. Box: 1641 59.072-970. \\ sc.ulhoa@gmail.com \\ J. F. da Rocha Neto \\ Instituto de Física, Universidade de Brasília 70910-900, \\ Brasilia, DF, Brazil. \\ rocha@fis.unb.br \\ J. W. Maluf \\ Instituto de Física, Universidade de Brasília 70904-970, \\ Brasilia, DF, Brazil. \\ P.O. Box: 04385 . \\ wadih@unb.br \\ Received Day Month Year \\ Revised Day Month Year \\ Communicated by Managing Editor
}

\begin{abstract}
We present a method to calculate the gravitational energy when asymptotic boundary conditions for the space-time are not given. This is the situation for most of the cosmological models. The expression for the gravitational energy is obtained in the context of the teleparallel equivalent of general relativity. We apply our method first to the Schwarzschild-de Sitter solution of Einstein's equation, and then to the RobertsonWalker Universe. We show that in the first case our method leads to an average energy density of the vacuum space-time, and in latter case the energy vanishes in the case of null curvature.
\end{abstract}

Keywords: Teleparallelism; Torsion Tensor; Cosmology.

\section{Introduction}

Recently some investigations have revived the question about the total energy of the Universe, including the material energy and the energy of the gravitational field \begin{tabular}{ll|l|l|l|l|l}
\hline & 2 & 3 & 4 & 5 & 6 & 7
\end{tabular} . This question is nontrivial because the usual treatment of the energy of a cosmological model with pseudotensors or Komar's integral does not seem appropriate, since the model is described by a metric that is not asymptotically flat 8. The problem holds even in an empty Universe described by de Sitter's solution, since in this case there is a (non-flat) cosmological horizon. 
The motivation for finding an expression for the energy of the universe comes from the fact that this subject may help to answer some questions about the origin and evolution of the universe, with consequent improvements for the cosmological models 5 .

Several investigations and results indicate that the total energy of the universe is zero. However these results are dubious since most of them are obtained by means of pseudotensors, which are coordinate-dependent expressions whose geometrical meaning is not clear. The results obtained by means of Komar's integrals, on the other hand, depend on the normalization of a Killing vector and in the case of cosmological systems, which are not asymptotically flat, there is no physically preferred choice for such vectors.

In the framework of the Teleparallel Equivalent of General Relativity (TEGR) the gravitational energy is well defined for finite volumes of the three dimensional space, and in particular for asymptotically flat space-times. When asymptotic boundary condition are not available, for instance in the case of the RobertsonWalker model and of the de Sitter Universe, a new method has to be used. As remarked by Faddeev 9, an expression for the gravitational energy must vanish only in the total absence of matter and gravitational field. Therefore in order to avoid the use of boundary conditions, it is possible, and perhaps mandatory, to use regularized expressions 10 , which do not require the establishment of asymptotic conditions. The purpose of this paper is to point out that the use of a regularized expression for the gravitational energy is quite suitable to cosmological models.

This paper is organized as follows. In section 2, we introduce the formalism of teleparallel gravity. We show how to define the gravitational energy in this context and present the definition of the regularized expression for the gravitational energy, which can be applied for arbitrary field configurations. In section 3, we propose a method to evaluate the energy based on the regularized expression, since in cosmology the lack of spatial asymptotic conditions prevents the application of ordinary methods. We evaluate the energy for two configurations which have relevance in cosmology, the Schwarzschild-de Sitter's solution and the Robertson-Walker model for the universe. Finally we present some concluding remarks.

Notation: space-time indices $\mu, \nu, \ldots$ and $\mathrm{SO}(3,1)$ indices $a, b, \ldots$ run from 0 to 3. Time and space indices are indicated according to $\mu=0, i, \quad a=(0)$, $(i)$. The tetrad field is denoted by $e^{a}{ }_{\mu}$, and the flat, Minkowski space-time metric tensor raises and lowers tetrad indices and is fixed by $\eta_{a b}=e_{a \mu} e_{b \nu} g^{\mu \nu}=(-1,+1,+1,+1)$. The determinant of the tetrad field is represented by $e=\operatorname{det}\left(e^{a}{ }_{\mu}\right)$ and we use the constants $G=c=1$.

\section{The regularized gravitational energy-momentum expression in TEGR}

The TEGR is an alternative formulation of the theory of general relativity, and 
is constructed out of tetrad fields in the Weitzenböck space-time of distant parallelism. In the Hamiltonian formulation the constraint equations are interpreted as energy, momentum and angular momentum equations for the gravitational field 11 . Two vectors are said to be parallel if their projections on the tangent space by means of the action of the tetrad field are equal. Thus, considering two vectors, $V^{a}(x)=e^{a}{ }_{\mu} V^{\mu}(x)$ and $V^{a}(x+d x)=e^{a}{ }_{\mu} V^{\mu}(x)+\left(e^{a}{ }_{\lambda} \partial_{\mu} V^{\lambda}+V^{\lambda} \partial_{\mu} e^{a}{ }_{\lambda}\right) d x^{\mu}=$ $V^{a}+e^{a}{ }_{\mu}\left(\nabla_{\lambda} V^{\mu}\right) d x^{\lambda}$, distant from each other by an infinitesimal displacement, the teleparallelism or distant parallelism is obtained if

$$
\nabla_{\nu} V^{\mu}=\partial_{\nu} V^{\mu}+\left(e^{a \mu} \partial_{\nu} e_{a \lambda}\right) V^{\lambda}=0
$$

where $e^{a \mu} \partial_{\nu} e_{a \lambda}=\Gamma_{\nu \lambda}^{\mu}$ is called the Weitzenböck connection. It is simple to check that $\nabla_{\mu} e^{a}{ }_{\lambda} \equiv 0$.

It is well known that in the Riemannian geometry the Christoffel symbols ${ }^{0} \Gamma_{\mu \nu}^{\lambda}$ are symmetric in the lower indices and the corresponding torsion tensor vanishes. However, the field equations in teleparallel gravity are constructed out of the torsion tensor $T^{\lambda}{ }_{\mu \nu}$ of the Weitzenböck connection, where $T^{\lambda}{ }_{\mu \nu}=e_{a}{ }^{\lambda} T^{a}{ }_{\mu \nu}$, and

$$
T^{a}{ }_{\mu \nu}(e)=\partial_{\mu} e^{a}{ }_{\nu}-\partial_{\nu} e^{a}{ }_{\mu} .
$$

The curvature tensor constructed out of the Weitzenböck connection $\Gamma_{\mu \nu}^{\lambda}=$ $e^{a \lambda} \partial_{\mu} e_{a \nu}$ vanishes identically.

Let ${ }^{0} \omega_{\mu a b}$ represent the torsion-free Levi-Civita connection,

$$
\begin{aligned}
{ }^{0} \omega_{\mu a b} & =-\frac{1}{2} e^{c}{ }_{\mu}\left(\Omega_{a b c}-\Omega_{b a c}-\Omega_{c a b}\right), \\
\Omega_{a b c} & =e_{a \nu}\left(e_{b}{ }^{\mu} \partial_{\mu} e_{c}{ }^{\nu}-e_{c}{ }^{\mu} \partial_{\mu} e_{b}{ }^{\nu}\right),
\end{aligned}
$$

The Christoffel symbols ${ }^{0} \Gamma_{\mu \nu}^{\lambda}$ and the Levi-Civita connection are identically related by $\partial_{\mu} e^{a}{ }_{\nu}+{ }^{0} \omega_{\mu}{ }_{b}{ }_{b} e^{b}{ }_{\nu}-{ }^{0} \Gamma_{\mu \nu}^{\lambda} e^{a}{ }_{\lambda}=0$, or

$$
{ }^{0} \Gamma_{\mu \nu}^{\lambda}=e^{a \lambda} \partial_{\mu} e_{a \nu}+e^{a \lambda}\left({ }^{0} \omega_{\mu a b}\right) e^{b}{ }_{\nu} .
$$

In the teleparallel equivalent of GR the following identity is relevant in the construction of the Lagrangian density,

$$
{ }^{0} \omega_{\mu a b}=-K_{\mu a b},
$$

where $K_{\mu a b}=\frac{1}{2} e_{a}{ }^{\lambda} e_{b}{ }^{\nu}\left(T_{\lambda \mu \nu}+T_{\nu \lambda \mu}+T_{\mu \lambda \nu}\right)$ is the contorsion tensor.

Let us first write the curvature scalar constructed out of (3). It is possible to show that such a quantity is given by

$$
e R\left({ }^{\circ} \omega\right) \equiv-e\left(\frac{1}{4} T^{a b c} T_{a b c}+\frac{1}{2} T^{a b c} T_{b a c}-T^{a} T_{a}\right)+2 \partial_{\mu}\left(e T^{\mu}\right) .
$$


where $e$ is the determinant of the tetrad field $e^{a}{ }_{\mu}$ and $T^{a}=T^{b}{ }_{b}{ }^{a}$. Both sides of (44) are invariant under Lorentz transformations. Dropping the divergence term we construct the Lagrangian density

$$
\begin{aligned}
\mathcal{L}\left(e_{a \mu}\right) & =-k e\left(\frac{1}{4} T^{a b c} T_{a b c}+\frac{1}{2} T^{a b c} T_{b a c}-T^{a} T_{a}-2 \Lambda\right)-\mathcal{L}_{M} \\
& \equiv-k e \Sigma^{a b c} T_{a b c}-\mathcal{L}_{M}+2 k e \Lambda
\end{aligned}
$$

where $k=1 /(16 \pi), \mathcal{L}_{M}$ stands for the Lagrangian density for the matter fields, $\Lambda$ is the cosmological constant and $\Sigma^{a b c}$ is defined by

$$
\Sigma^{a b c}=\frac{1}{4}\left(T^{a b c}+T^{b a c}-T^{c a b}\right)+\frac{1}{2}\left(\eta^{a c} T^{b}-\eta^{a b} T^{c}\right) .
$$

Performing the variational derivative of the Lagrangian density with respect to the tetrad field $e_{a \lambda}$ we get, after some rearrangements, the field equation

$$
\partial_{\nu}\left(e \Sigma^{a \lambda \nu}\right)=\frac{1}{4 k} e e^{a}{ }_{\mu}\left(t^{\lambda \mu}+T^{\lambda \mu}\right)+\frac{1}{2} \Lambda e e^{a \lambda},
$$

where

$$
t^{\lambda \mu}=k\left(4 \Sigma^{b c \lambda} T_{b c}{ }^{\mu}-g^{\lambda \mu} \Sigma^{b c d} T_{b c d}\right),
$$

and $e^{a}{ }_{\mu} T^{\lambda \mu}=\frac{1}{e} \frac{\delta \mathcal{L}_{M}}{\delta e_{a \lambda}}$ corresponds to the energy-momentum tensor of matter fields. It is possible to show, by explicit calculations, that (7) is equivalent to the Einstein's field equations with the cosmological term.

Now let us analyze the meaning of $t^{\lambda \mu}$. In view of the ant-symmetry property $\Sigma^{a \mu \nu}=-\Sigma^{a \nu \mu}$ it follows that

$$
\partial_{\lambda}\left[e e^{a}{ }_{\mu}\left(t^{\lambda \mu}+T^{\prime \lambda \mu}\right)\right]=0,
$$

which is a local balance equation, where $T^{\prime \lambda \mu}=T^{\lambda \mu}+2 k \Lambda g^{\lambda \mu}$. Therefore we identify $t^{\lambda \mu}$ as the gravitational energy-momentum tensor 12 .

The integration of $t^{\lambda \mu}+T^{\prime \lambda \mu}$ over a hyper-surface $x^{0}=$ constant defines the energy-momentum vector due to gravitational and matter fields,

$$
P^{a}=\int_{V} d^{3} x e e^{a}{ }_{\mu}\left(t^{0 \mu}+T^{\prime 0 \mu}\right),
$$

where $V$ is a volume of the three-dimensional space. Since the integrand is a scalar density under coordinate transformations, $V$ may be finite as well as infinite (in which case $S$ is finite or $S \rightarrow \infty$, respectively). In view of the field equations, eq. (10) may be written as 


$$
P^{a}=-\int_{V} d^{3} x \partial_{j} \Pi^{a j}=-\oint_{S} d S_{j} \Pi^{a j},
$$

where $\Pi^{a j}=-4 k e \Sigma^{a 0 j}$. It is interesting to note that the above expression is invariant under coordinate transformations of the three-dimensional space, under time reparametrizations, and is projected on the tangent space which means that it is coordinate independent. We point out that the definition above was first presented in Ref. 13 .

In this formalism gravitation is considered as a manifestation of torsion. Therefore in the flat space-time the torsion tensor is supposed to vanish. However this con-

dition in some cases is not satisfied 10 , and in this case we have to adopt regularized expressions. A regularized expression for the gravitational energy-momentum 10 is defined by

$$
P_{r e g}^{a}=-\int_{V} d^{3} x \partial_{k}\left[\Pi^{a k}(e)-\Pi^{a k}(E)\right],
$$

where $\Pi^{a j}(E)$ is constructed out of flat tetrads $E^{a}{ }_{\mu}$ which are obtained from $e^{a}{ }_{\mu}$ by requiring the vanishing of the physical parameters. This definition guarantees that the energy-momentum of the flat space-time always vanishes. In the context of the energy problem in general relativity, the idea and concept of a regularized expression was first considered by Brown and York $14 \mid 15$ in the realm of the quasilocal definition of the gravitational energy. Conceptually, our approach regarding the regularization does not differ from that considered by these authors.

In eq. (12) $e^{a}{ }_{\mu}$ and $E^{a}{ }_{\mu}$ are separately solutions of Einstein's equations. The flat tetrads $E^{a}{ }_{\mu}$ may yield nonvanishing values for the tensor $T^{a}{ }_{\mu \nu}$, and for this reason the regularization procedure is necessary. Note that definition (10) follows from Einstein's equations, and a similar equation holds for $E^{a}{ }_{\mu}$. Thus eq. (12) may be understood as the subtraction of the two Einstein's equations for $e^{a}{ }_{\mu}$ and $E^{a}{ }_{\mu}$. We remark, however, that in view of eq. (4) a local $\mathrm{SO}(3,1)$ transformation of the tetrad field yields only a surface term in the Lagrangian density (5). The surface term does not contribute to the field equations, which are unaffected by a local $\mathrm{SO}(3,1)$ transformation of the tetrad fields.

Tetrad fields that in the flat space-time limit reduce to unit vectors in the $r$, $\theta$ and $\phi$ directions yield nonvanishing torsion tensor components 10. The great advantage of using such tetrad fields is that they do not require the prescription of boundary conditions. This is precisely the case in cosmology. However, they require the use of a regularization procedure.

\section{The Procedure to Evaluate the Gravitational Energy}

For any solution of Einstein's field equations, such as the Schwarzschild or Kerr solutions, the choice of the tetrad field fixes the frame adapted to a certain observer 
in space-time. Such a choice may be motivated, for instance, by the asymptotic conditions of the tetrad at spatial infinity, in which case we take the tetrad field to represent the flat space-time in this limit. However, in cosmology this is not the usual situation, since one does not require boundary conditions for the metric tensor as one does in the analysis of localized material systems in gravitation. Instead of boundary conditions, in cosmology one usually requires the concept of homogeneity of the three-dimensional space, in agreement with the cosmological principle, which asserts that we do not occupy any special position in the universe. The concept of isotropy of space is an additional requirement of the cosmological models. Thus in cosmology we consider the tetrad field to represent a local basis for an arbitrary observer in the chosen coordinate system, and make use of the regularization procedure. Let us analyze the procedure in the following cases.

\subsection{The Schwarzschild-de Sitter Space-Time}

The most general spherically symmetric vacuum solution of the field equations with a positive cosmological constant $\Lambda$ is the Schwarzschild-de Sitter solution,

$$
d s^{2}=-\left(1-\frac{2 m}{r}-\frac{r^{2}}{R^{2}}\right) d t^{2}+\left(1-\frac{2 m}{r}-\frac{r^{2}}{R^{2}}\right)^{-1} d r^{2}+r^{2} d \theta^{2}+r^{2} \sin ^{2} \theta d \phi^{2},
$$

where $R=\sqrt{\frac{3}{\Lambda}}$. This metric specifies the de Sitter vacuum solution when we set $m=0$, and the Schwarzschild solution when $\Lambda=0$.

Let us choose a diagonal tetrad field like

$$
e_{\mu}^{a}(t, r, \theta, \phi)=\left(\begin{array}{cccc}
A & 0 & 0 & 0 \\
0 & A^{-1} & 0 & 0 \\
0 & 0 & r & 0 \\
0 & 0 & 0 & r \sin \theta
\end{array}\right),
$$

where $A=\left(1-\frac{2 m}{r}-\frac{r^{2}}{R^{2}}\right)^{1 / 2}$. The determinant $e$ of (14) reads $e=r^{2} \sin \theta$. In terms of one-forms the spatial components are given by $e^{(1)}=A^{-1} d r, e^{(2)}=r d \theta$ and $e^{(3)}=r \sin \theta d \phi$. The spatial components of the tetrad field represent unit vectors in the $r, \theta$ and $\phi$ directions. Therefore such a tetrad field is interpreted as the usual basis in spherical coordinates for local observers.

In order to calculate the energy of the configuration defined by (13), we have to evaluate the component $\Sigma^{001}$, obtained from (6), which after algebraic manipulations is given by

$$
\Sigma^{001}=\frac{1}{2} g^{00} g^{11}\left(g^{22} T_{212}+g^{33} T_{313}\right),
$$

where the components of torsion tensor appearing in the above expression are 


$$
\begin{aligned}
& T_{212}=r, \\
& T_{313}=r \sin ^{2} \theta .
\end{aligned}
$$

Thus integrating over a surface of constant radius $r_{0}$ in (11) it follows that

$$
\begin{aligned}
P^{(0)} & =4 k \oint_{S} d S_{1}\left(e \Sigma^{001}\right) \\
& =\lim _{r \rightarrow r_{0}}\left(-r \sqrt{-g_{00}}\right) .
\end{aligned}
$$

However, using the regularized expression given by eq. (12), we find that the energy contained within a surface of constant radius $r_{0}$ is

$$
P_{\text {reg }}^{(0)}=r_{0}\left(1-\sqrt{1-\frac{2 m}{r_{0}}-\frac{r_{0}^{2}}{R^{2}}}\right)
$$

Let us evaluate expression (18) for the range of values of $r_{0}$ such that

$$
\frac{2 m}{r_{0}} \ll 1, \frac{r_{0}^{2}}{R^{2}} \ll 1 .
$$

The above conditions mean that the cosmological constant is very small. Expanding (18) and neglecting all powers of both $\frac{2 m}{r_{0}}$ and $\frac{r_{0}{ }^{2}}{R^{2}}$ we arrive at

$$
P_{r e g}^{(0)}=m+\frac{r_{0}^{3}}{6} \Lambda \text {. }
$$

which yields, for $\Lambda=0$, the well known value of the energy of the Schwarzschild space-time.

Now we will instead consider the vacuum de Sitter solution only, since in this case the main features are not altered by the introduction of a mass $m$ at $r=0$. Let us assign $E_{d S}$ as the value of the energy in the absence of the mass $m$, which is the background (vacuum) energy.

The total gravitational energy $E_{d S}$ contained in the physical region of the vacuum de Sitter space is obtained from (18) by making $m=0$ and $r_{0}=R$. It follows that

$$
E_{d S}=R=\sqrt{\frac{3}{\Lambda}} .
$$

If we substitute $r=R \sin \chi$ in (13), then we shall have a space where the coordinate $\chi$ varies from 0 to $\pi$. The surface of a sphere in these coordinates is $S=4 \pi R^{2} \sin ^{2} \chi$, which increase reaching its maximum value $4 \pi R^{2}$ at $\chi=\pi / 2$, after which it decrease 
to zero at $\chi=\pi 16$. The volume is $V=\int_{0}^{\pi} d \chi 4 \pi R^{3} \sin ^{2} \chi$ therefore the mean energy density is given by

$$
\frac{E_{d S}}{V}=\frac{E_{d S}}{2 \pi^{2} R^{3}}=\frac{\Lambda}{6 \pi^{2}} .
$$

Thus when we consider an Universe filled with matter, the accelerated rate of expansion of the Universe, which is usually explained by the hypothesis of the so called dark energy, may be due to the mean energy density of the vacuum spacetime. Expression (21) already appeared in Ref. 17, however we decide to reconsider the same problem since in the present procedure we do not specify any boundary conditions for the tetrad field. This method is better suited to describe cosmological models.

\subsection{The Homogeneous and Isotropic Universe}

The cosmological principle asserts that the large-scale structure of the Universe reveals homogeneity and isotropy 18 . The most general form of a line element that preserves such features may be written as 16 ,

$$
d s^{2}=-d t^{2}+a^{2}(t)\left[\frac{d r^{2}}{1-k^{\prime} r^{2}}+r^{2}\left(d \theta^{2}+\sin ^{2} \theta d \phi^{2}\right)\right],
$$

where $a(t)=S(t) /|K|^{1 / 2}$ if $K \neq 0$ and $a(t)=S(t)$ if $K=0 . S(t)$ is the scale factor and $K$ is the constant curvature of space. Here $K=|K| k^{\prime}$ where $k^{\prime}$ assumes the values $+1,0,-1$ which correspond to (i) a three-space of constant positive curvature, (ii) a flat space or (iii) a space of constant negative curvature, respectively. By introducing a new radial parameter $\bar{r}$ related to $r$ by

$$
r=\frac{\bar{r}}{1+k^{\prime} \bar{r}^{2} / 4}
$$

we can write the line element above as

$$
d s^{2}=-d t^{2}+\frac{[a(t)]^{2}}{\left(1+k^{\prime} \bar{r}^{2} / 4\right)^{2}}\left(d \bar{r}^{2}+\bar{r}^{2}\left(d \theta^{2}+\sin ^{2} \theta d \phi^{2}\right)\right) .
$$

This second form is called the Roberston-Walker (RW) line element. In the following, we will consider the first line element. The quantity $a(t)$ is obtained as a solution of the Friedman's equations,

$$
\begin{aligned}
& 3 \frac{\dot{a}^{2}}{a^{2}}+3 \frac{k^{\prime}}{a^{2}}=8 \pi \rho, \\
& \frac{\dot{a}^{2}}{a^{2}}+2 \frac{\ddot{a}}{a^{2}}+\frac{k^{\prime}}{a^{2}}=-8 \pi p,
\end{aligned}
$$

where $\rho$ and $p$ denote the density of the cosmological medium and its pressure. 
In what follows we will show that in the context of the TEGR it is possible to arrive at an expression for the gravitational energy of the universe for closed, open and flat cases, respectively, using the following diagonal tetrad field,

$$
e_{\mu}^{a}(t, r, \theta, \phi)=\left(\begin{array}{cccc}
1 & 0 & 0 & 0 \\
0 & A & 0 & 0 \\
0 & 0 & r a(t) & 0 \\
0 & 0 & 0 & r a(t) \sin \theta
\end{array}\right),
$$

where $A=\frac{a(t)}{\sqrt{\left(1-k^{\prime} r^{2}\right)}}$. The determinant $e$ of (27) reads $e=A a^{2}(t) r^{2} \sin \theta$.

In order to obtain the energy of this system, we have to calculate $\Sigma^{001}$, which reads

$$
\Sigma^{001}=\frac{1}{2} g^{00} g^{11}\left(g^{22} T_{212}+g^{33} T_{313}\right)
$$

where

$$
\begin{aligned}
& T_{212}=r a^{2}(t), \\
& T_{313}=r a^{2}(t) \sin ^{2} \theta .
\end{aligned}
$$

Taking into account eq. (11), we integrate now expression (28) over a twodimensional spacelike surface $\mathrm{S}$ defined by a radius $r=r_{0}=$ constant. We arrive at,

$$
\begin{aligned}
P^{(0)} & =4 k \oint_{S} d S_{1}\left(e \Sigma^{001}\right) \\
& =-a r_{0} \sqrt{1-k^{\prime}\left(r_{0}\right)^{2}} .
\end{aligned}
$$

We apply now eq. (12), and after the regularization procedure we obtain

$$
P_{r e g}^{(0)}=\operatorname{ar}_{0}\left(1-\sqrt{1-k^{\prime}\left(r_{0}\right)^{2}}\right) .
$$

The expression (31) was obtained in Ref. ${ }^{19}$ by means of a totally different method. This fact supports our own procedure and result.

In the case of flat space-time $k^{\prime}=0$, the energy is zero. This result is consistent with the fact that in this case we have an open (infinit) space with null curvature. This means that in this configuration, the gravitational energy is minus the energy of the fields of matter for any value of volume considered. In this configuration, the gravitational energy and the energy of matter fields exactly cancel out and the resultant space is flat.

For the case of $k^{\prime}=1$ we have a closed space with constant positive curvature, and the total energy given by eq. (31) for an arbitrary volume of space is always 
positive and linear in the scale factor. This result reveals the fact that, in this case, the gravitational energy and the energy of the matter fields do not exactly cancel out, and the difference is likely to be responsible for the nonvanishing positive curvature.

Finally in the case of space with constant negative curvature $k^{\prime}=-1$ (open space) we can see from eq. (31) that the resultant energy (gravitational energy plus the energy of matter fields) is always negative for any value of volume, in particular it is infinite negative when $r_{0} \rightarrow \infty$. Here it is important to note that in this case we have an infinite space with constant negative curvature. It is likely that in this case, the total negative energy, is responsible for the constant negative curvature of the space.

Recently 20 negative energy was obtained for cosmological models describing open universes. In 20 all 9 types of Bianchi homogeneous cosmological models has been examined by using an energy expression obtained out of a Hamiltonian boundary term in the context of tetrad-teleparallel gravity. In contrast to our results, the authors have found zero energy for some non-flat cosmological models. However for space with negative curvature the results in 20 have the same sign of our result for space with negative curvature. As stated in 20 , for the case $k^{\prime}=-1$, each region of constant negative curvature acts like a concave lens exactly as if it had a negative matter density repelling light rays from these.

\section{Conclusion}

It is well known that solutions of Einstein's equations that describe cosmological models do not have well defined boundary conditions in the same way as the configurations of isolated sources. The absence of such conditions in these configurations is a difficulty for the determination of a definite expression of gravitational energy for cosmological models. In this work we have proposed a coordinate independent method to investigate the energy of gravitational field configurations when asymptotically flat conditions are not available. The definition given by eq. (12) in the absence of matter fields gives the gravitational energy, and in the presence of matter fields gives the total energy.

We applied our method to the homogeneous and isotropic Universe and to the Schwarzschild-de Sitter solution. When the cosmological constant is set to zero in the latter case, it is well known that the space-time is asymptotically flat. When the cosmological constant is nonvanishing, the cosmological horizon prevents the existence of such boundary (asymptotic) conditions. When one tries to use the tetrad field as a local frame such that the spatial components are unit vectors along the $r, \theta$ and $\phi$ directions, which is realized by means of a diagonal tetrad of the type given by eq. (14), some drawbacks may arise. For example, the emergence of non-vanishing torsion components in Minkowski space-time. These drawbacks are eliminated by means of the regularization procedure, which is similar to that given in the work by Brown and York $\frac{15}{}$ for quasi-local energy expression. The regularization 
procedure is powerful because it dispenses the use of boundary conditions for the gravitational field.

\section{References}

1. Nathan Rosen. The energy of the universe. General Relativity and Gravitation, 26(3):319-321, 1994.

2. N. Banerjee and S. Sen. Einstein pseudotensor and total energy of the universe. Pramana, 49(6):609-615, 1997.

3. T. Vargas. The energy of the universe in teleparallel gravity. Gen. Rel. Grav., 36:1255, 2004.

4. F. I. Cooperstock and M. Israelit. The energy of the Universe. Foundations of Physics, 25:631-635, April 1995.

5. F. I. Cooperstock. The energy of the Universe. Gen. Rel. Grav., 26(3):323-327, 1994.

6. S. S. Xulu. Total Energy of the Bianchi Type I Universes. Int. J. Theor. Phys., 39:1153-1161, 2000.

7. Lszl B. Szabados. Quasi-local energy-momentum and angular momentum in general relativity. Living Reviews in Relativity, 12(4), 2009.

8. Valerio Faraoni and F. I. Cooperstock. On the total energy of open FriedmannRobertson-Walker universes. Astrophys. J., 587:483-486, 2003.

9. L. D. Faddeev. The energy problem in einstein's theory of gravitation (dedicated to the memory of v. a. fock). Soviet Physics Uspekhi, 25(3):130-142, 1982.

10. J. W. Maluf, M. V. O. Veiga, and J. F. da Rocha-Neto. Regularized expression for the gravitational energy- momentum in teleparallel gravity and the principle of equivalence. Gen. Rel. Grav., 39:227-240, 2007.

11. J. W. Maluf, S. C. Ulhoa, F. F. Faria, and J. F. da Rocha-Neto. The angular momentum of the gravitational field and the Poincare group. Class. Quant. Grav., 23:62456256, 2006.

12. J. W. Maluf. The gravitational energy-momentum tensor and the gravitational pressure. Annalen Phys., 14:723-732, 2005.

13. J. W. Maluf and J. F. da Rocha-Neto. General Relativity on a Null Surface: Hamiltonian Formulation in the Teleparallel Geometry. Gen. Rel. Grav., 31(2):173-185, 1999.

14. J. D. Brown and Jr. J. W. York. Quasi-local energy in general relativity. In J. E. Marsden M. J. Gotay and V. Moncrief, editors, Proceedings of the Joint Summer Research Conference on Mathematical Aspects of Classical Field Theory. American Mathematical Society, 1991.

15. J. David Brown and James W. York. Quasilocal energy and conserved charges derived from the gravitational action. Phys. Rev. D, 47(4):1407-1419, Feb 1993.

16. Ray d'Inverno. Introducing Einstein's Relativity. Clarendon Press, Oxford, 4th edition, 1996.

17. J. W. Maluf and J. F. da Rocha-Neto. The vacuum energy density in the teleparallel equivalent of general relativity. Arxiv: gr-qc/0308084, 2003.

18. L. D. Landau and E. M. Lifshiz. The Classical Theory of Fields, volume 2 of Course of Theoretical Physics. Elsevier Butterworth-Heinemann, 4th revised english edition, 2004.

19. C. Chen, J. Liu, and J. M. Nester. Quasi-Local Energy for Cosmological Models. Mod. Phys. Lett. A, 22:2039-2046, 2007.

20. James M. Nester, Lau Loi So, and T. Vargas. Energy of homogeneous cosmologies. Phys. Rev. D, 78(4):044035, Aug 2008. 